J. Lake Sci. (湖泊科学), 2007, 19(3):345-350

http:// www. jlakes. org. E-mail: jlakes@ niglas. ac.cn

(c) 2007 by Journal of Lake Sciences

\title{
太湖鲫鱼数量变化的规律及与环境间关系的分析”
}

\author{
刘恩生 ${ }^{1,2}$, 刘 正文 ${ }^{2}$,鲍传和 ${ }^{1}$ \\ $(1:$ 安徽农业大学动物科技学院, 合肥 230036$)$ \\ (2: 中国科学院南京地理与湖泊研究所,南京 210008)
}

摘 要: 通过食物组成分析和渔获物组成调查, 并结合历年渔获量统计和环境监测资料, 研究了太湖鲫鱼数量变化的规 律、机制及与环境间的相互关系. 渔获物调查和渔获量统计资料均表明: 近年鲫鱼数量不断增加, 已成为太湖仅次于鲚鱼 的重要鱼类. 2004 年渔获物调查中,鲫鱼占鱼类总重量的 $8.40 \% \pm 2.69 \%$,占鲤、鲫鱼渔获量的 $65.08 \% \pm 15.47 \%$; 历年 渔获量统计显示, $1952-1998$ 年鲤、鲫鱼渔获量相对稳定在 $1010.1 \pm 367.2$ 、占鱼类总量 $8.89 \% \pm 5.33 \%, 1999$ 年上升达 到 $2547.4 \mathrm{t}$ 、占 $9.45 \%, 2000$ 年达到 $3708.7 \mathrm{t}$, 占 $12.18 \%$; 年龄组成分析表明,鲫鱼低龄化趋势在加剧. 食性分析表明:在 7-12 月, 微囊藻占鲫鱼食物体积的 83.65\%-93.99\%. 分析认为:太湖鲫鱼以微囊藻为主要食物; 富营养化加重为其提 供了充足覀料, 实施禁渔期、禁渔区等措施使其繁殖得到保障, 捕捞强度过高导致其年龄组成低龄化、个体小型化. 而太 湖鲫鱼以蓝藻为主要食物, 可能对蓝藻有抑制作用.

关键词: 太湖; 鲫鱼; 渔获量; 年龄组成; 食物组成

\section{The changes of catches of Carassius auratus and relationship with environment in Lake Taihu, China}

\author{
LIU Ensheng ${ }^{1,2}$, LIU Zhengwen ${ }^{2} \&$ BAO Chuanhe ${ }^{1}$ \\ ( 1 :Anhui Agicultural University, Hefei 230036, P. R. China) \\ (2: Nanjing Institute of Geography and Limnology, CAS, Nanjing 210008, P. R. China)
}

\begin{abstract}
Based on the analysis of food content and the survey of the composition in fish catches and the data of fish catches in Lake Taihu, the amount changes of catches of Carassius auratus Linnaeus and Their Mutual Relationship with Environment were studied. The results of the survey of fish composition in 2004 and the statistics data of fish catches from 1952 to 2004 showed that: Within recent years, the catches of Carassius auratus Linnaeus had been increasing and had become the second dominant fishes following Coilia ectenes taihuensis Yen et Lin. The analysis of age structure showed that the percentage of $0^{+}$fishes increased and the average fish body size decreased. For example, the percentage of $0^{+}$Carassius auratus Linnaeus had been increasing according to the trend of $y=$ 19. $154 \mathrm{e}^{0.0566 x}$ ( $y$ means the percentage of $0^{+}$fishes $\%, x$ means time a). And the average body length had been decreasing according to the trend of $y=115.45 \mathrm{e}^{-0.0312 x}$ ( $y$ means body size $\mathrm{cm}, x$ means time a). The results of food content analysis showed that: the percentage of blue-algae accounted for about $83.65 \%-93.99 \%$ of food volume of Carassius auratus Linnaeus. Under the conditions of eutrophication in Lake Taihu, Carassius auratus Linnaeus mainly fed on blue-algae; eutrophication resulted in the increasing of blue-algae which could be eaten by the fish. The measures of protecting fishes were carried out to ensure the fish propagating. In addition, the trend of percentage of low age fishes increasing and the trend of body size of the fishes decreasing were caused by over-fishing. Carassius auratus Linnaeus might restrain blue-algae increasing because it mainly fed on blue-algae.
\end{abstract}

Keywords: Lake Taihu; Carassius auratus; fish catches; age structure; food content 
鲫鱼 (Carassius auratus Linnaeus) 是我国湖泊常见的定居性鱼类. 因其可在湖泊繁殖、食性广、对环境适 应能力强而成为湖泊渔业的主要增殖对象, 产量一般较高. 有关鲫鱼的生物学研究及增殖技术有很多报 道 $^{[1-5]}$. 随着湖泊富营养化程度的日趋加重,鱼类对环境下行效应的研究也已受到国内外重视. 国外的大 量研究认为, 鲤、鲫鱼通过提高水柱氮磷含量使藻类和浮游生物食性鱼类产量增加. 如 Andersson $\mathrm{G}$ 等发 现, 底栖鱼类通过摄食摚动沉积物和消化活动中的磷释放, 大大增加了水柱的氮磷量, 从而使藻类生物量升 高 $^{[6]}$; Richardson 等认为鲤鱼使底栖无脊椎动物生物量大大降低、初级生产力增加 ${ }^{[7]}$; 而 Northcote $\mathrm{T} \mathrm{G}$ 的工 作发现鲤鱼提高了藻类和鲢鱼的产量 ${ }^{[8]}$. 我国学者在武汉东湖的研究也发现, 鲤鱼加速了底泥中磷的释 放,并通过 “短路代谢” 提供营养, 对浮游植物的生长起促进作用 ${ }^{[9]}$. 但总体看, 我国有关鲤、鲫鱼和环境间 相互关系的研究还较少, 因此有必要对此进行研究.

在太湖,鲤、鲫鱼渔获量是合并统计的. 近年鲤、鲫鱼渔获量有快速增加趋势, 如 2000 年达到 $3708.7 \mathrm{t}$, 占鱼类总量的 $12.18 \%$ 。而 2004 年调查鲫鱼占鲤、鲫鱼渔获量的 $65.08 \% \pm 15.47 \%$, 已成为太湖仅次于鲚 鱼的重要鱼类. 有关太湖鲫鱼的食性等生物学内容虽早有研究 ${ }^{[10-13]}$, 但鱼类的食性是随环境的变化而变 化的,而在富营养化条件下太湖鲫鱼食性的相应变化以及和环境间相互关系的研究未见报道. 为此, 通过 $2004-2005$ 年对太湖鲫鱼占鱼类组成比例、年龄结构及食物组成的分析, 并结合历年渔获量统计和环境监 测资料,研究了太湖鲫鱼数量变化的规律、机制以及和环境间的相互影响.

\section{1 材料和方法}

\section{1 鲫鱼占鱼类组成比例和历年渔获量}

鲫鱼占鱼类组成比例的调查于 2004 年 9 月 - 10 月 15 日在三山湖、焦山、贡湖、平台山和宜兴滩 5 个水 域进行 (图 1). 捕捞工具为高踏网, 网长 $2550 \mathrm{~m}$ 、网目 $\mathrm{a}=5 \mathrm{~mm}$, 每次捕捞水域面积折合约 $52 \mathrm{hm}^{2}$. 按常规 方法进行鱼类生物学资料采集和统计. 对 16 个网次、共计 $74432.1 \mathrm{~kg}$ 渔获物全部分类称重,主要计算鲤、 鲫鱼在渔获物中的重量组成比例. 又结合当年全湖渔获量统计进行鱼类组成比例分析. 鲤、鲫鱼历年渔获 量根据太湖渔业生产管理委员会 $1952-2004$ 年的统计年报 ${ }^{[14]}$,太湖鲤、鲫鱼和鳊鱼是合并统计的,但鳊鱼 数量很少,因此,鲤、鲫鱼、鳊鱼的历年渔获量基本可以看作鲤鱼、鲫鱼的渔获量.

\section{2 年龄组成比例}

年龄组成分析是根据篓网渔获物进行的. 篓网网长一般为 $200-250 \mathrm{~m}$ 、网目为 $\mathrm{a}=10 \mathrm{~mm}$. 对 2004 年 9 月 1 日至 12 月 15 日期间共计 1752 尾鲫鱼进行了年龄组成分析. 采样地点在三山湖、焦山和宜兴滩 3 个水 域进行 (图 1). 篓网的选择性较小,其渔获物的年龄组成是可以代表湖泊中鲫鱼年龄组成实际情况的.

年龄鉴定根据鳞片:取胸鯺下方、侧线上方鳞片,用 $5 \%$ 氨水洗净后置于载玻片上,用低倍显微镜根据 年轮鉴别 ${ }^{[15,16]}$. 共鉴定 $0^{+}$鲫鱼 41 尾, $1^{+}$及以上鲫鱼 31 尾, 根据各龄组平均体长对 1752 尾鲫鱼样品分类 计数后计算年龄组成比例.

\section{3 食物组成分析}

食物组成分析于 2005 年 6 月 15 日开始. 整个期间可以分为 6 月 15 日到 8 月 30 日的非捕捞期间和 9 月 1 日到 12 月 30 日的捕捞期间. 非捕捞期间采样布点在三山湖、焦山和宜兴滩 3 个水域附近采样; 捕捞期 间在三山湖、焦山、贡湖、平台山和宜兴滩 5 个水域采样(图 1 ).

我国学者在分析鱼类食物组成时多用出现率这一指标. 出现率可以反映鱼类对某种饵料的喜好程度, 但并不能反映某种饵料占食物的比例及具体的数量 ${ }^{[17,18]}$. 作者对太湖鲚鱼的食性研究已发现, 出现率指标 会高估次要食物的重要性 ${ }^{[19]}$. 因此,在分析鱼类的食物组成时,用数量、重量以及体积比例作为指标. 在研 究中发现, 鲫鱼食物中几乎全部是微囊藻. 在分析食物组成的体积比例时采用视野体积计数法: 把消化道 中的全部食物稀释至 $50-100 \mathrm{ml}$, 混合均匀后用 $0.1 \mathrm{ml}$ 浮游生物计数框全部计数, 微囊藻则以群体计数. 计数时显微镜的目镜用 $10 \times$ 、物镜用 $40 \times$. 对 7-8 月份的鲫鱼样本系根据视野粗略估计; 对 $9-12$ 月份的 鲫鱼样本计算了出现率、数量组成比例,并根据数量组成比例折算为体积组成比例. 体积的折算是根据《湖 泊生态调查观测与分析》中浮游植物、浮游动物细胞体积表 ${ }^{[16]}$ 并结合测微尺实际测量. 每个食物样本计数 3 次, 求出平均数. 


\section{2 结果}

\section{1 鲫鱼组成比例及数量变化}

于 2004 年 9 月 -10 月 15 日,对三山湖、焦山、贡 湖、平台山和宜兴滩 5 个水域的高踏网渔获物全部分 类称重,主要计算鲫鱼在鱼类组成中的比例. 结果表 明, 鲫鱼占鱼类总重量的 $8.40 \% \pm 2.69 \%$ (表 1). 按 此推算, 在鲤、鲫鱼产量中, 鲤鱼占 $34.92 \pm 15.47 \%$, 鲫鱼占 $65.08 \pm 15.47 \%$.

从渔获量统计资料看, $1952-2004$ 年的 53 年里, 太湖鲤、鲫鱼渔获量的变化大致可分为两个段段: $1952-1998$ 年的 47 年间, 渔获量相对稳定在 1010.1 $\pm 367.2 \mathrm{t}$, 占鱼类总量的 $8.89 \% \pm 5.33 \% ; 1999$ 年开 始上升达到 $2547.4 \mathrm{t}$ 、占 9.45\%, $2000-2002$ 年达到 $3500-3700 \mathrm{t}$, 占总量的 $11.7 \%-14.7 \% ; 2003-2004$ 年两年产量略有下降, 但仍达到 $2748 \mathrm{t}$ 和 $2788 \mathrm{t}$, 占总 渔获量的 $9.23 \%$ 和 $8.39 \%$ (图 2). 渔获量统计资料 和本次调查的鱼类组成比例结果是基本一致的, 说明

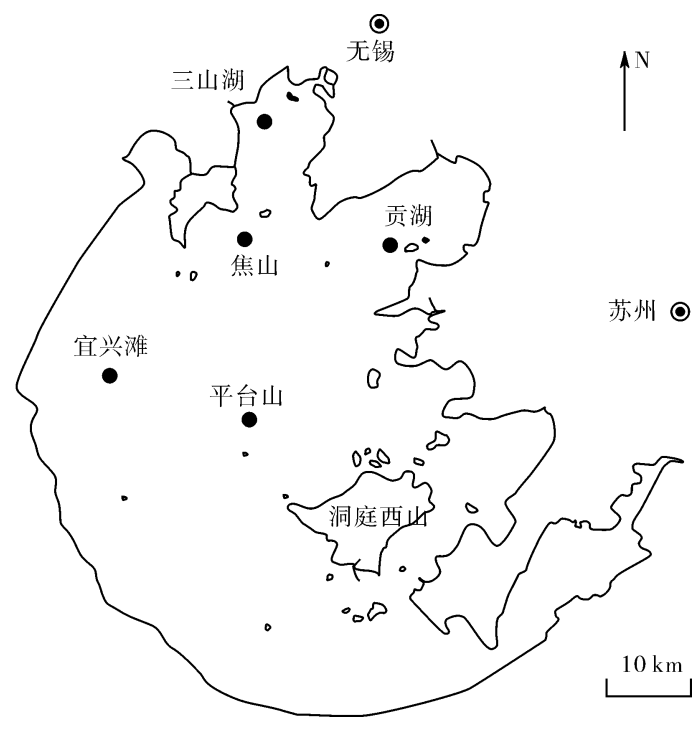

图 1 太湖采样点分布

Fig. 1 Sampling points in Lake Taihu 在太湖近年鲫已成为仅次于鲚鱼的重要鱼类.

\section{2 年龄结构及生长}

于 2004 年 9 月 - 10 月 15 日,对 1752 尾鲫鱼分析了年龄组成比例. 结果表明, $0^{+}$鲫鱼数量比例为 $91.72 \% \pm 2.76 \%$ 、重量比例为 $79.11 \% \pm 1.97 \%, 1^{+}$及以上鲫鱼数量比例仅占 $8.28 \% \pm 1.52 \%$ 、重量比例 为 $20.89 \% \pm 0.86 \% .0^{+}$鲫鱼平均体重为 $38.9 \mathrm{~g}$, 渔获物平均体重为 $45.1 \mathrm{~g}$ (表 2). 鲫鱼体长、体重回归公 式为 $W=0.076 L^{2.5958}\left(R^{2}=0.9515 、 n=107\right)$. 式中 $W$ 为体重 $(\mathrm{g}), L$ 为体长 $(\mathrm{cm})$ (图 3$)$.

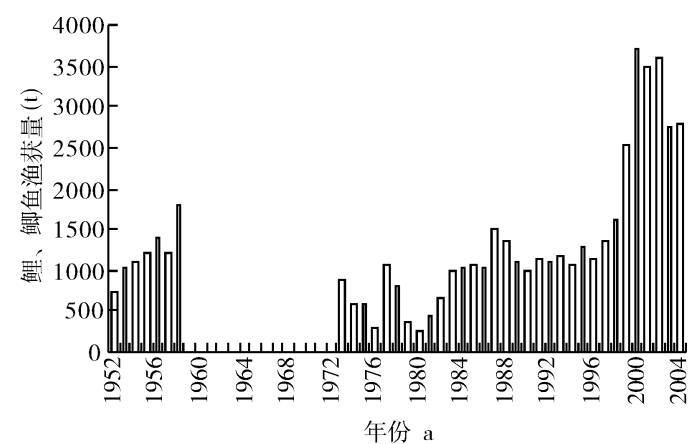

图 $21952-2004$ 年太湖鲤、鲫鱼渔获量变化趋势

Fig. 2 changes of Cyprinus carpio Linnaeus and

Carassius auratus Linnaeus catches from 1952 to 2004

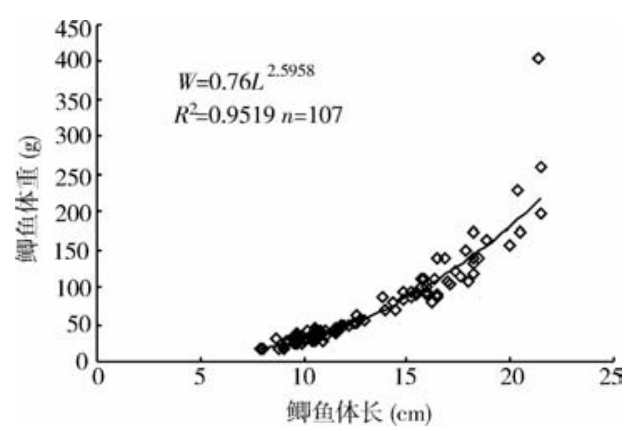

图 3 鲫鱼体长 - 体重间的关系

Fig. 3 the relationship between weight and body length of Carassius auratus Linnaeus

和历史资料比较发现,鲫鱼年龄组成的低龄化和小型化趋势明显. 如 1979、1981、1982 和 1989 年太湖 鲫鱼渔获物的平均体重分别为 $118.77 \mathrm{~g} 、 99.01 \mathrm{~g} 、 87.56 \mathrm{~g}$ 和 $94.90 \mathrm{~g}$; 渔获物中 $0^{+}$鲫鱼的数量比分别为 $20.5 \% 、 22.37 \% 、 29.45 \%$ 和 $26.56 \%$ (1). 太湖鲫鱼 $0^{+}$个体的数量比是按照 $y=19.154 \mathrm{e}^{0.0566 x}\left(y\right.$ 为鲫鱼 $0^{+}$个 体比例 $(\%), x$ 为时间 (a)) 趋势在增加; 鲫鱼平均体长是按照 $y=115.45 \mathrm{e}^{-0.0312 x}(y$ 为鲫鱼平均体长 
$(\mathrm{cm}), x$ 为时间 (a)) 的趋势在逐渐减小 (图 4、5).

表 1 鲫鱼在鱼类组成中的重量比例\%

Tab. 1 percentage of Carassius auratus Linnaeus weight accounting for total fish catches

\begin{tabular}{cccccc}
\hline 种类 & 鲚鱼 & 鲤鱼 & 鲫鱼 & 间䰻 & 其它鱼类 \\
\hline 重量比 $\%$ & $84.04 \pm 4.07$ & $4.68 \pm 2.51$ & $8.40 \pm 2.69$ & $1.60 \pm 1.52$ & $1.12 \pm 0.72$ \\
\hline
\end{tabular}

表 2 鲫鱼年龄组成数量比和重量比 $(\%)$

Tab. 2 Percentage of amount and weight of Carassius auratus Linnaeus in total fish catches

\begin{tabular}{ccccccc}
\hline 龄组 & 尾数 & 尾数比 $(\%)$ & 重量 $(\mathrm{kg})$ & \multicolumn{1}{c}{ 重量比 $(\%)$} & 平均尾重 $(\mathrm{g})$ 总平均尾重 $(\mathrm{g})$ \\
\hline $0^{+}$ & 1607 & $91.72 \pm 2.76$ & 62.5 & $79.11 \pm 1.97$ & 38.9 & 45.1 \\
$1^{+}$及以上 & 145 & $8.28 \pm 1.52$ & 16.5 & $20.89 \pm 0.86$ & 113.8 & \\
\hline
\end{tabular}

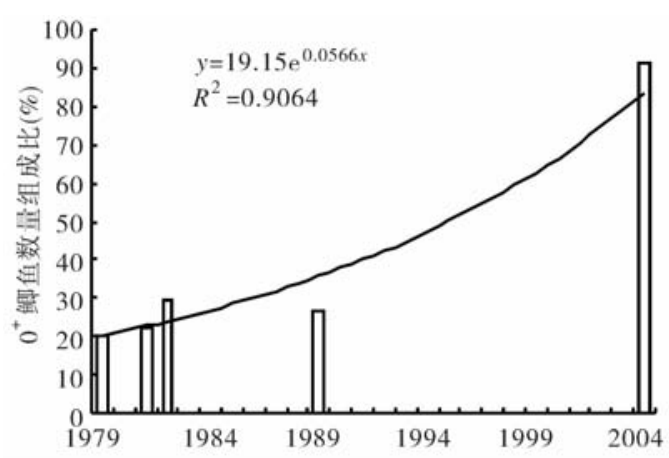

图 $40^{+}$鲫鱼数量组成比历年变化趋势

Fig. 4 changes of amount percentage of

$0^{+}$Carassius auratus Linnaeus

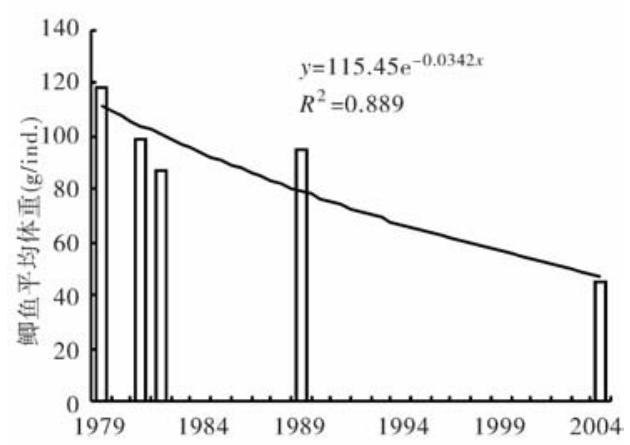

图 5 鲫鱼种群平均体重历年变化趋势 Fig. 5 changes of the average body length of Carassius auratus Linnaeus

\section{3 食物组成}

调查结果表明:在 7-8 月份, 微囊藻约占鲫鱼食物食物体积的 $85 \%-90 \%$, 颤藻约占 $10 \%$, 水生植物 碎片约占 $2 \%-7 \%$, 枝角类和轮虫合计约占 $1 \%-2 \%$. 其中微囊藻和颤藻合计占 $95 \%$ 以上(表 3 ). $9-12$ 月份, 微囊藻占鲫鱼食物数量的 $93.10 \% \pm 4.05 \%$, 折算体积比为 $93.99 \% \pm 3.34 \%$, 而浮游动物约占数量 的 $7.06 \% \pm 4.05 \%$, 折算体积比为 $6.01 \% \pm 3.34 \%$.

表 3 鲫鱼食物组成

Tab. 3 food content of Carassius auratus Linnaeus during July-Aug. and Sept. -Dec.

\begin{tabular}{ccccc}
\hline $7-8$ 月 & 微囊藻 & 㗜藻 & 象鼻溞、轮虫、浮游动物碎片水生植物碎片 \\
出现率 $(\%)$ & 100 & 100 & 80.43 & 50.00 \\
估计体积比 $(\%)$ & $83.65 \pm 7.07$ & $10.48 \pm 2.86$ & $0.97 \pm 0.91$ & $2.71 \pm 5.39$ \\
\hline $9-12$ 月 & 微囊藻 & 象鼻溞 & 其它枝角类碎片 & 轮虫 \\
出现率 $(\%)$ & 100 & 95.56 & 73.33 & 6.67 \\
数量比 $(\%)$ & $93.10 \pm 4.05$ & $6.47 \pm 3.42$ & $0.35 \pm 1.06$ & $0.08 \pm 0.47$ \\
折算体积比 $(\%)$ & $93.99 \pm 3.34$ & $5.59 \pm 2.99$ & $0.42 \pm 0.93$ & $0.09 \pm 0.41$ \\
\hline
\end{tabular}




\section{3 讨论}

\section{1 太湖富营养化条件下鲫鱼的食性变化}

结果表明, 在 7-12 月份鲫鱼的食物中均以微囊藻为主. 鱼类的食性是因环境而变化的. 在富营养化 条件下, 太湖初级生产力几乎全部是由蓝藻所形成. 而从微囊藻群体形成的颗粒大小和数量看, 是鲫鱼容 易得到的食物. 虽然目前尚不知鲫鱼对微囊藻的消化利用能力,但食物中仅出现的少量浮游动物明显不足 以维持其生长和正常生命活动. 这可能间接说明, 在其主要生长季节里, 维持其生命活动的能量来源几乎 完全是微囊藻. 这和通常认为的鲫鱼主要摄食底栖植物和动物的情况是不同的. 在湖泊渔业生产中, 鲫鱼 渔产力的估计通常是以底栖生物的数量为计算根据, 这明显和太湖的实际情况不符合. 对太湖鲫鱼渔产力 的估计应该根据已经发生变化的食性特点,研究在湖泊富营养化条件下鲫鱼利用蓝藻的生产能力.

\section{2 鲫鱼数量变化的原因}

分析表明, 太湖近年来鲫鱼产量不断增加的主要原因是:富营养化加重为鲫鱼提供了充足饵料; 捕食鲫 鱼的肉食性鱼类数量较少;禁渔期、禁渔区等措施的实施使其繁殖得到保障.

在鲤、鲫鱼产量中, 鲤鱼占 $34.92 \% \pm 15.47 \%$, 鲫鱼占 $65.08 \% \pm 15.47 \%$. 而食性分析证明, 鲫鱼是以 微囊藻为主要食物的. 随着太湖富营养化程度的不断加重, 经常的蓝藻暴发为鲫鱼提供了最容易得到的食 物来源. 此外,太湖鲤、鲫鱼产量的增加和 COD 的不断升高基本一致(图 6).

太湖的主要肉食性鱼类是尧嘴鲌和蒙古鲌,其产量合并统计为鲌鱼. 而近年来太湖鲌鱼产量不断降 低, 仅维持在 $100 \mathrm{t}$ 左右的捕捞产量. 并且, 许品诚对翘嘴鲌的食性分析表明, 食物中并没有鲤、鲫鱼出 现 ${ }^{[20]}$. 作者曾经研究过湖泊中摄食鲤、鲫鱼的主要肉食性鱼类是乌鳢 ${ }^{[21]}$ 和鲭鱼, 而在太湖这两种鱼类的数 量较少, 没有形成可以捕捞的产量. 因此, 可以认为, 在太湖能够大量捕食鲤、鲫鱼的肉食性鱼类是很少的. 这会使当年鲤、鲫鱼的存活率较高.

有关太湖鱼类资源繁殖保护效果的分析表明 ${ }^{[14]}$, 鲤、鲫鱼产量变化趋势和繁殖保护措施的实施是一致 的. 如在 20 世纪 60 年代末至 70 年代初,在我国内陆水域掀起 “围湖造田”运动, 根据统计,在太湖被围剭 的水面达到 $1.33 \times 10^{4} \mathrm{hm}^{2}$. 被围怎的水面通常是水草茂盛、草上产卵鱼类的良好繁殖场所. 因此,在这一 期间鲤、鲫鱼产量较低,如 1976 年仅 $276.3 \mathrm{t}$ (图 2). 由于产卵场所被破坏,在较长时间里很难快速恢复, 鲤、鲫鱼产量在 $1973-1983$ 年期间始终处于较低水平, 平均年产量仅 $630 \mathrm{t}$, 比 $1952-1958$ 年期间下降了 47.6\%.1984 年后开始实施半年封湖休渔, 较好地促进了鲤、鲫鱼增殖. 如 1984-1992 年平均产量又恢复 到 $1000 \mathrm{t}$ 以上(图 2). 1987 年后, 太湖禁渔期在不断延长, 平均达到 $200 \mathrm{~d}$ 以上. 水生植被面积开始恢复、禁 渔区范围也在不断扩大. 鲤、鲫鱼增殖和育肥条件不断改善,这也是其产量增加的重要原因.

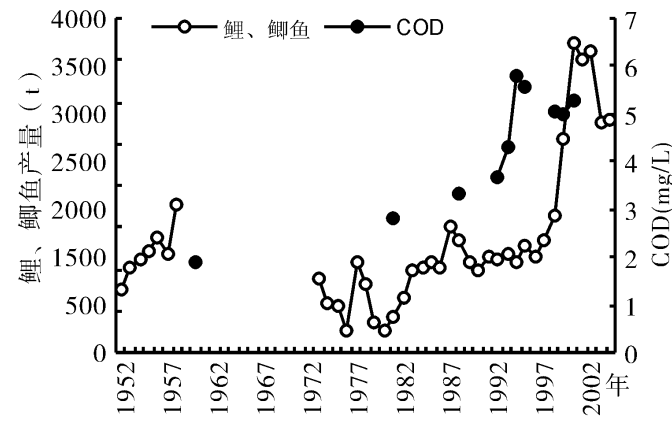

图 6 鲤、鲫鱼渔获量和 COD 历年变化趋势比较

Fig. 6 changes of COD and catches of Cyprinus carpio

Linnaeus and Carassius auratus Linnaeus

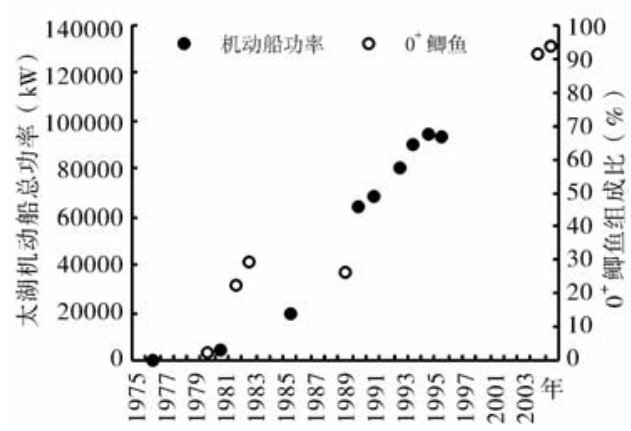

图 7 太湖机动船总功率和 $0^{+}$鲫鱼数量组成比历年变化比较 Fig. 7 changes of the total power of machine boat and the amount percentage of Cyprinus carpio Linnaeus and Carassius auratus Linnaeus

\section{3 鲫鱼年龄组成变化的原因}

分析太湖鱼类捕捞强度的变化可以发现, 鲫鱼种群的低龄化趋势与捕捞强度的不断提高是一致的. 太 
湖的捕捞强度在不断升高, 如按照捕捞机动船的功率计算, 1980 年比 1975 年增加了 13.25 倍; 1985 年比 1980 年增加了 4 倍; 到 1990 年, 比 1985 年增加了 3.3 倍, 1995 年又比 1990 年增加了 1.5 倍 (1). 由于捕捞强 度很高, 这会使高龄鱼比例减少; 而太湖摄食鲫鱼的肉食性鱼类数量很少, 这又使当年繁殖的幼鱼成活率较 高. 因此,太湖鲫鱼年龄组成的特点是当年鱼占 $90 \%$ 以上, 并且低龄化有继续加强趋势 (图 7 ).

\section{4 参考文献}

［1］殷名称. 鱼类生态学. 北京:中国农业出版社,1995:64.

[2] 孙兴旺. 淇河鲫的生物学特征. 淡水渔业, 1986,(2):5-8.

３］崔悦礼. 滇池高背鲫的种群变异和利用问题. 淡水渔业,1987,(3):27.

[4] 华元渝, 张 建, 陈亚芬. 滆湖鲫、鲤鱼的增产潜力及渔业生态管理研究. 长江流域资源与环境, 1996,5(3):236 - 241 .

[5] 雷惠僧等. 池塘养鱼学. 上海: 上海科学技术出版社, 1981:5-30.

[6] Andersson G et al. Effects of planktivorous and benthivorous fish on organisms and water chemistry in eutrophic lakes. Hydrobiologia. 1978, $59: 9-15$.

[7] Richardson W B et al. Food web response to the experimental manipulation of a benthivore (Cyprinus carpio), zooplanktivore (Menidia beryllina) and benthic insects. Arch. Hydrobiol, 1990, 119:143 - 165.

[8] Northcote IG. Fish in the structure and function of freshwater ecosystems: a "top-down” view. Can J Fish Aquat Sci, 1988, $361-379$.

[9] 刘建康主编. 东湖生态学研究 (二). 北京:科学出版社, 1990:117.

[10] 朱成德. 太湖水产资源的合理利用与增殖途径的初步探讨. 淡水渔业, 1982,(4):40-42.

[11] 朱成德,钟宣世. 太湖人工放流效果的初步探讨. 淡水渔业, 1978,(2):2-9.

[12] 顾良伟. 太湖人工放流的初步探讨. 水产学报, 1986,10(2):223-228.

[13] 朱成德,钟宣世. 太湖人工放流效果的初步探讨. 淡水渔业, 1984,(5):2-7.

[14] 倪 勇,朱成德主编. 太湖鱼类志. 上海: 上海科学技术出版社,2005:26-28;46-48;217-218.

[15] 唐 渝. 太湖湖鲚生长特征和临界年龄的研究. 生态学杂志, 1986,5(3):5-9.

[16] 黄祥飞,陈伟民,蔡启铭. 湖泊生态调查观测与分析. 北京: 中国标准出版社, 1999 .

[17] 孟田湘. 渤海重要底层鱼类食物重叠系数与鱼类增殖. 海洋水产研究, 1989,10:1 - 7 .

[18] 薛 芗,金显仕.鱼类食性和食物网研究评述. 海洋水产研究, 2003, 24(2):76-87.

[19] 刘恩生,刘正文,陈伟民等. 太湖鲚鱼渔获量变化及与生物环境间相关关系的研究. 湖泊科学, 2005,17 (4) $: 340-345$.

[20] 许品诚. 太湖尧嘴红白的生物学及其增殖问题的探讨. 水产学报, 1984,8(4):279.

[21] 刘恩生,郑玉林, 江 河. 对乌鳢在花园湖渔业开发中生态地位初析. 水产学报. 1992, 16(2): 147 - 153 . 